\title{
Evaluación del costo de producción para faenas de aserrío portátil
}

Production cost evaluation in portable bandsaw mill operations

\author{
ALFREDO AGUILERA ${ }^{1}$, LUIS INZUNZA ${ }^{1}$, ROSA ALZAMORA ${ }^{2}$, LUIS TAPIA ${ }^{3}$. \\ ${ }^{1}$ Universidad Austral de Chile, Instituto de Tecnología de Productos Forestales, Casilla 567, \\ Valdivia, Chile. E-mail: aguilera@uach.cl, linzunza@uach.cl \\ ${ }^{2}$ Universidad Austral de Chile, Instituto de Manejo Forestal, Casilla 567, Valdivia, Chile. \\ E-mail: ralzamora@uach.cl \\ ${ }^{3}$ Ingeniero Forestal
}

\begin{abstract}
SUMMARY
New technologies in portable bandsaw mill operations for small and medium size production scale shows several advantages face to traditional saw practice in the country. The main importance aspects are better yield of raw material, lowness surface roughness of sawn timber, great equipment mobility and versatility in cutting programs. However, production costs involved in each operation is a fundamental aspect to consider, because determine his feasibility. The study developed shows a detailed production costs analysis in different scenarios, for bandsaw mill operations, reflecting consistent results in computer program developed for this study, concerning technical and economical feasibility in real operations.
\end{abstract}

Key words: bandsaw, cut, quality, costs.

\section{RESUMEN}

La introducción de nuevas tecnologías en las faenas de aserrío, para instalaciones de pequeña y mediana escala, presenta numerosas ventajas respecto de los métodos tradicionalmente utilizados en el país, principalmente en aspectos de mayor aprovechamiento de las trozas, mejor calidad de terminación, movilidad de los equipos y versatilidad en los programas de corte. Sin embargo, un aspecto fundamental en la correcta operación de la faena son, sin duda, los costos asociados a ella, que determinarán la factibilidad de su ejecución. El estudio desarrollado presenta un análisis detallado de los costos de producción en diferentes escenarios, para faenas de aserrío portátil con una máquina de sierra hincha, reflejando la validez del programa computacional elaborado para el estudio de factibilidad técnicoeconómico en situaciones reales de trabajo.

Palabras clave: sierra huincha, corte, calidad, costos.

\section{INTRODUCCION}

El aprovechamiento racional de los recursos forestales juega un rol importante en el desarrollo económico y social del país. Reducir la pérdida de materias primas, mejorar la calidad de los productos y optimizar el uso, tanto de la mano de obra como de los equipos, son una tarea pendiente $y$ en continuo desarrollo en este sector productivo.

El impacto de los pequeños productores de madera aserrada, tanto a nivel nacional como regional, es significativo sobre la economía del sector, estando éstos sujetos a problemas de estacio- 
nalidad de la producción, bajos rendimientos, altos costos y mala calidad de los productos, por citar algunos aspectos.

Los aserraderos móviles producen actualmente cerca del $42 \%$ de la madera aserrada nativa del país, de los cuales cerca de 500 instalaciones que presentan un bajo nivel tecnológico se encuentran paralizadas (1), siendo los altos costos de instalación, el elevado número de personal y el bajo aprovechamiento del trozo, los principales motivos del cierre de estas instalaciones. Es necesario, además, tener en cuenta que la rentabilidad de la inversión se verá reflejada fuertemente por los volúmenes disponibles, por las exigencias del mercado en términos de dimensiones correctas de las piezas, por la calidad de los productos, precios razonables y productos alternativos.

Nuevas tecnologías implementadas en el sector forestal maderero nacional permiten mejorar la rentabilidad mediante el aumento de los rendimientos, la optimización de la producción y la reducción de los costos de operación. Entre estas nuevas tecnologías para el aserrío móvil se encuentran los equipos con sierra huincha horizontal, que, además, presentan características tales como su fácil transporte, personal limitado, buenas terminaciones superficiales de la madera producida, exactitud de las medidas, tiempos reducidos de instalación y un alto aprovechamiento del trozo. Dada su capacidad de ser fácilmente transportable, estos equipos pueden ser empleados en operaciones de zonas remotas con mala accesibilidad.

Los aserraderos portátiles generalmente se instalan en un lugar remoto por un período de tiempo restringido. Según esto, la localización seleccionada para un aserradero portátil debe ser tal, que requiera el mínimo de gastos previos de preparación para su utilización, tanto en dinero como en tiempo (2).

La capacidad de estos aserraderos de trasladarse fácilmente de un lugar a otro permite una economía en los costos de materia prima; además, es posible obtener un margen de utilidad superior en relación a una instalación fija. El alto aprovechamiento del trozo es una de las ventajas más importantes $\mathrm{y}$, a veces, descuidadas en los equipos portátiles de sierra huincha.

La calidad y precisión de los productos obtenidos con los equipos portátiles huincha es alta, proporcionando una terminación superficial más uniforme y lisa en relación a un aserradero circular. Siendo, además, la efectividad del proceso mucho mayor que este último tipo de instalación.

La efectividad del proceso de aserrío (conversión) se expresa comúnmente como el rendimiento obtenido de la troza, es decir, la cantidad de madera aserrada neta respecto del volumen bruto del trozo. Según Somerville (3), la efectividad del proceso de conversión está estrechamente relacionada con el tipo de aserradero. Para Cown (4), también influyen el tamaño y curvatura del trozo.

Se debe elegir el método de corte que produzca el máximo retorno para cubrir los gastos, por lo que es importante obtener el óptimo aprovechamiento por escuadría. Además, considerar el precio de mercado de la madera rolliza puesta en el aserradero y los costos del proceso, para mantener en lo posible una baja producción de productos secundarios que se encuentren fuera de las escuadrías programadas (5).

Es importante considerar que el costo de la materia prima representa un $70 \%$ del costo total de manufactura de la madera aserrada. Cuando el costo de los trozos aumenta, los aserraderos deben tomar todas las medidas necesarias para incrementar el rendimiento del proceso (6).

Los planes de corte tienen entonces una importancia vital sobre la utilización de los trozos, ya que para cada clase diamétrica se determina un diagrama de corte óptimo, según el tipo de producto a obtener. De ellos dependen las pérdidas de madera que se producen en la forma de aserrín, lampazos u otros. Estos planes de corte dependen del tipo de maquinaria y los defectos que presente la materia prima con que cuenta el aserradero (7).

En la práctica se ha detectado una deficiencia importante en la evaluación preliminar para las operaciones de estos equipos. Es decir, no existe una cuantificación precisa de todos los costos presentes en la operación de una faena determinada, por un control inadecuado tanto de los tiempos muertos como del detalle de todos los costos involucrados en el proceso, desconociendo, entonces, el productor cuál será el nivel real de rentabilidad de su negocio para una situación precisa. Por esto, el objetivo general de este estudio es el de desarrollar un simulador de costos de aserrío portátil que permita el procesamiento de la información y la generación de informes de salida. En sus objetivos específicos pretende entregar una 
herramienta que permita: (a) determinar los costos fijos y variables de la operación, (b) determinar los costos por unidad de volumen y de tiempo, (c) comparar costos en forma rápida y segura, de diferentes faenas, y (d) ejecutar un análisis de sensibilidad frente a cambios en el costo de algunos insumos de la producción.

\section{MATERIAL Y METODOS}

Se realizó un estudio de tiempo y rendimiento para una faena de aserrío con la especie "tepa" (Laurelia philippiana Looser). Se utilizaron un aserradero huincha portátil marca Wood-Mizer modelo LT 30HD y una sierra canteadora. Con la información recopilada en terreno en la obtención de dos tipos de producto denominados "P1" y "P2" se desarrolló una planilla Excel que permite determinar los costos de producción.

Durante las fases de desarrollo del sistema de análisis se contempló el estudio del tamaño de muestra, el cual permitió definir para el producto P1 (piezas de 1 pulgada de espesor) y producto P2 (2 pulgadas de espesor) un tamaño de muestra de 52 trozos, los cuales arrojaron un volumen de 1.240 pulgadas aserradas. Para ello se controlaron 52 ciclos de trabajo, donde cada ciclo fue fraccionado en 5 momentos. En total se realizaron 208 momentos, claramente diferenciados en el proceso de aserrío, analizándose independientemente uno del otro.

El estudio fue desarrollado con el método de tiempo continuo, donde se controló el tiempo de cada momento, definido dentro del ciclo de trabajo. Entonces el ciclo del estudio de tiempo se caracterizó de la siguiente manera $(8,9)$ :

Momento A: movimiento del trozo en bote.

Momento B: acomodo del trozo.

Momento C: corte.

Momento D: ordenamiento de la madera.

Momento E: trasporte.

Posteriormente se realizó la determinación del volumen total de cada troza utilizando la regla maderera de Casimiro Donat y la Norma JAS. Con esta información se determinó el rendimiento del equipo, el cual se ve asociado en forma directa con los costos.

En relación a las variables financieras, se consideraron aquellas relacionadas con los equipos, la tasa de interés de mercado, el valor de compra y el valor de salvamento. Asimismo, también la depreciación, el costo de la inversión media anual, los costos horarios, impuestos, costos de mantención, insumos y variables de producción personal, como los bonos de producción.

El programa computacional generado como resultado de este estudio (denominado ASEPORT), para el tratamiento y análisis de los datos obtenidos en terreno, contempla en el primero de sus bloques el ingreso de la información, es decir, las variables generales, la definición del volumen, las variables financieras de los equipos, accesorios del personal, sueldos, bonos, mantención de equipos y el consumo de energía. Los resultados son entregados en la forma de variables iniciales de cálculo, costos accesorios del personal, costos de reparaciones, costos de mantención, costos de combustible, costos variables totales, costos fijos totales y costos totales.

En relación a las variables generales, éstas establecen algunas variables que son relevantes en los cálculos posteriores:

- Contenido de cada tipo de producto en el volumen total (P1 y P2): se debe especificar el porcentaje en que se encuentran contenidos cada uno de los dos productos a obtener.

- Jornadas planificadas: corresponde a los días planificados mensuales estipulados por contrato.

- Jornadas perdidas: corresponde a los días perdidos por traslados, desperfectos mecánicos, etc. Estos reducen la producción y aumentan los costos.

- Productividad: señala el nivel de eficiencia en el uso del tiempo en las faenas. Si se desea trabajar con tiempos planificados se descarta la productividad.

- Producción horaria: se especifica la producción por hora para los productos determinados.

Importante a considerar es la definición del volumen, el cual es un parámetro esencial para la estimación de los costos. Se consideran tres alternativas en el ingreso del volumen: (a) Modalidad aproximación, la que corresponde cuando se estimen los parámetros generales de los trozos desde los cuales se cubicará el volumen total de todos los trozos existentes; (b) Modalidad volumen conocido: corresponde al ingreso ya sea del total de pulgadas cubicadas con Donat o bien en $\mathrm{m}^{3}$ JAS 
para que el programa cubique; (c) Modalidad inventario: esta corresponde al ingreso de la información en la forma de un inventario realizado en cancha.

\section{RESULTADOS Y DISCUSION}

Tratamiento de los costos. Variables iniciales de cálculo: el simulador ASEPORT muestra en un inicio todas las variables que son relevantes en el procesamiento posterior de la información de la faena, las cuales están reflejadas en el cuadro 1.

\section{CUADRO 1}

Variables iniciales de cálculo para la faena en estudio.

Initial variables of calculate for the task in study.

\begin{tabular}{|lrl|}
\hline Itemes & Valor & Unidad \\
\hline Volumen total & 24.200 & p.m./faena \\
\hline Escuadría & & \\
P1 & 12.100 & p.m./faena \\
P2 & 12.100 & p.m./faena \\
\hline Jornadas efectivas de producción & & \\
\% productividad & $100 \%$ & \\
horas productivas & 8 & hpr/jorn \\
jornadas productivas efectivas (JPE) & 24 & jor/mes \\
jornadas efectivas totales (JET) & 21 & jor/mes \\
\hline Rendimiento & & \\
P1 & 35,25 & p.m./hpl \\
P2 & 40,46 & p.m./hpl \\
\hline Cálculo de jornadas para completar faena & \\
Jornadas efectivas término & & \\
de faena (JTF) & 80,29 & jor/faena \\
Meses por faena & & \\
según JPE (MJPE) & 3,35 & meses/faena \\
Meses por faena & & \\
según JPE + pérdidas (MJET) & 3,82 & meses/faena \\
Producción mensual (PM) & $6.329,52$ & p.m./mes \\
\hline
\end{tabular}

Nota: $\mathrm{hpl}=$ hora planificada, $\mathrm{hpr}=$ hora productiva, p.m. = pulgada maderera.

\footnotetext{
${ }^{1}$ Valor del dólar a enero 2001 \$561.
}

Este cuadro resumen define la totalidad de información de rendimientos hora, productividad y volumen para una determinada faena, repercutiendo esta información directamente en los costos finales.

Se determinaron también los costos de los accesorios del personal, los costos de reparaciones los que están representados por un porcentaje derivado de la depreciación de los equipos, y los costos de mantención.

En el caso de los costos de reparaciones para el total de los equipos, este fue de 1,10 US\$(1)/ hora productiva (hpr), y los costos de mantención de 0,35 US\$/hpr para el aserradero, 0,29 US\$/hpr para la canteadora y $0,10 \mathrm{US} \$ / \mathrm{hpr}$ para el equipo de afilado.

Un ítem relevante lo constituye el combustible, dado que es un insumo esencial para el funcionamiento del aserradero. Los resultados del análisis se presentan en el cuadro 2 .

Se obtiene enseguida un cuadro resumen de los costos variables totales para la faena en estudio, los cuales se ven influenciados directamente por el nivel de producción (cuadro 3).

En relación a los costos fijos totales, el simulador pone a disposición del usuario un desglose completo de todos los ítemes involucrados, los cuales no se ven influenciados por el nivel de producción, estos se presentan en el cuadro 4.

\section{CUADRO 2}

Costos unitarios combustible y energía.

Unit costs of fuel and energy.

\begin{tabular}{|lrrl|}
\hline \multicolumn{4}{|c|}{ Costos de combustible y energía ${ }^{1}$} \\
\hline Combustible aserradero & Consumo & $1.927,00$ & 1/faena \\
& Costo & $1.339,60$ & US\$/faena \\
\hline Combustible canteadora & Consumo & 321,20 & 1/faena \\
& Costo & 223,27 & US\$/faena \\
\hline Equipo electrógeno & Consumo & 80,30 & 1/faena \\
& Costo & 55,82 & US\$/faena \\
\hline Vehículo & Consumo & 368,00 & 1/faena \\
& Costo & 255,83 & US\$/faena \\
\hline Otros equipos & Consumo & 35,65 & US\$/mes \\
& Costo & 119,27 & US\$/faena \\
\hline Electricidad (afilado) & Consumo & 17,83 & US\$/mes \\
& Costo & 59,63 & US\$/faena \\
\hline Costo total energía y combustible & $2.053,42$ & US\$/faena \\
\hline
\end{tabular}


BOSQUE 26(2): 107-114, 2005

Evaluación del costo de producción para faenas de aserrío portátil

\section{CUADRO 3}

Resumen costos variables unitarios estimados por ASEPORT.

Summary of unitary variable costs considered by ASEPORT.

\begin{tabular}{|lccrr|}
\hline & \multicolumn{4}{c|}{ Unidad } \\
\cline { 2 - 5 } Itemes & US\$/hpl & US\$/pulg & US\$/m3 & US\$/faena \\
\hline Energía y combustible & 3,20 & 0,08 & 3,58 & $2.053,42$ \\
Bonos & 2,55 & 0,07 & 2,86 & $1.639,22$ \\
Accesorios, corte y afilado & 0,85 & 0,02 & 0,95 & 546,41 \\
Costo reparación & 1,10 & 0,03 & 1,23 & 707,01 \\
Costo mantención & 1,05 & 0,03 & 1,18 & 676,53 \\
Total costos variables & 8,75 & 0,23 & 9,80 & $5.622,58$ \\
\hline
\end{tabular}

Nota: hpl = hora planificada

\section{CUADRO 4}

Resumen de costos fijos unitarios. Summary of unitary fixed costs.

\begin{tabular}{|lr|}
\hline Maquinaria & US\$/faena \\
\hline Depreciación & $1.795,59$ \\
Costo de la inversión media anual & $1.350,33$ \\
Seguros & 157,02 \\
Impuestos & 52,34 \\
Subtotal maquinaria & $3.355,28$ \\
Personal & $4.620,92$ \\
& \\
Total costos fijos & $7.976,20$ \\
\hline
\end{tabular}

Los costos totales de la faena, según unidad, con y sin considerar los costos fijos de depreciación y costo de la inversión media anual se presentan en el cuadro 5.

Estudio de tiempo. El estudio de tiempo entrega la posibilidad de tener un resumen general del desglose de los tiempos y porcentaje de cada uno de los momentos en que se fraccionó la operación de aserrío en un día de trabajo común, el cual se muestra en el cuadro 6.

Tiempo no productivo: son aquellos que, si bien son considerados tiempos planificados en el proceso productivo, no están contribuyendo en la producción. Estos se dividen en mantención pro-

\section{CUADRO 5}

Resumen de costos totales unitarios.

Summary of unitary total costs.

\begin{tabular}{|c|c|c|c|c|}
\hline \multicolumn{5}{|c|}{ Considerando la totalidad de costos fijos y variables } \\
\hline & US\$/hpl & US\$/pulg & $\mathrm{US} \$ / \mathrm{m}^{3}$ & US\$/faena \\
\hline Total costos fijos & 10,87 & 0,29 & 12,16 & $7.976,20$ \\
\hline Total costos variables & 8,75 & 0,23 & 9,80 & $5.622,58$ \\
\hline Total & 19,62 & 0,52 & 21,96 & $13.598,78$ \\
\hline \multicolumn{5}{|c|}{ Sin considerar costos fijos de depreciación ni costo de la inversión media anual } \\
\hline Total costos fijos & 6,58 & 0,17 & 7,36 & $4.830,29$ \\
\hline Total costos variables & 8,75 & 0,23 & 9,80 & $5.622,58$ \\
\hline Total & 15,33 & 0,40 & 17,16 & $10.452,87$ \\
\hline
\end{tabular}

Nota: $\mathrm{hpl}=$ hora planificada 
BOSQUE 26(2): 107-114, 2005

Evaluación del costo de producción para faenas de aserrío portátil

\section{CUADRO 6}

Tiempos promedio por trozo de los momentos dentro del ciclo de trabajo, incluyendo tiempos productivos y no productivos para 1 y 2 pulgadas

$$
\text { de espesor de la pieza. }
$$

Average time by log of the moments within the cycle of work, including productive and nonproductive times for 1 and 2 inches of thickness of the piece.

\begin{tabular}{|lrrrr|}
\hline & $\begin{array}{c}\text { Pieza de 1 pulgada } \\
\text { de espesor }\end{array}$ & \multicolumn{2}{c|}{$\begin{array}{c}\text { Pieza de 2 pulgadas } \\
\text { de espesor }\end{array}$} \\
\hline & Tiempo (min) & $\%$ & Tiempo (min) & $\%$ \\
\hline Tiempo no productivo & & & & \\
Mantención programada & $01: 31$ & 3,80 & $01: 49$ & 5,28 \\
Mantención no programada & $02: 23$ & 6,08 & $01: 52$ & 5,44 \\
Varios & $00: 30$ & 1,27 & $00: 49$ & 2,31 \\
Subtotal & $04: 24$ & 11,26 & $04: 31$ & 13,08 \\
& & & & \\
Tiempo productivo & & & & \\
Movimiento & $02: 50$ & 4,67 & $01: 08$ & 3,31 \\
Acomodación & $02: 41$ & 6,82 & $02: 23$ & 7,38 \\
Corte & $25: 04$ & 65,17 & $22: 09$ & 64,12 \\
Ordenar & $01: 58$ & 5,08 & $01: 41$ & 4,86 \\
Traslado & $02: 44$ & 6,94 & $02: 27$ & 7,21 \\
Subtotal & $35: 06$ & 88,73 & $30: 02$ & 86,96 \\
& & & & \\
Tiempo total & $39: 32$ & 100 & $34: 33$ & 100 \\
\hline
\end{tabular}

gramada (lubricación, limpieza), mantención no programada (cambios de huincha) y varios.

Tiempo productivo: es todo el tiempo que contribuye a la producción y está constituido por todos aquellos momentos del ciclo de trabajo programado, es decir, tiempo en movimiento de trozos, acomodación, corte, ordenamiento y traslado para comenzar otro ciclo.

El ítem "varios" dentro del tiempo no productivo es un tiempo que no presenta ninguna ganancia en producción. Las pérdidas detectadas en este ítem correspondieron principalmente al momento de cambio de huincha, donde se observó un promedio de 3 a 7 cambios diarios.

Dentro del ciclo de trabajo productivo, el momento de corte representa el mayor porcentaje de tiempo. Éste puede ser mejorado actuando sobre variables como método de aserrío, manejo de trozos, rapidez en el retiro de las piezas aserradas, calidad del afilado de huinchas y potencia del motor. Estas dos últimas variables influyen direc- tamente en la velocidad de corte y en la calidad de la madera terminada.

Rendimientos. El estudio de tiempo es la base para determinar el rendimiento del aserrío. Sobre éste derivan todos los costos y planificaciones que existen para una faena determinada. Es así como el promedio de dos días de trabajo para cada espesor fueron de 35,35 pulg/hora planificada en 1 pulgada de espesor y de 40,96 pulg/hora planificada para 2 pulgadas de espesor (cuadro 7).

Según Biaggio (10), el mayor rendimiento de madera aserrada es producida en las clases diamétricas superiores, las cuales no sólo involucran un aumento del valor de las trozas por su mayor volumen producido, sino que aumentan su valor potencial debido al ancho de las piezas producidas.

De esta manera al comparar los rendimientos promedios por clase diamétrica, para una escuadría determinada, se pudo apreciar que existe un mayor rendimiento al aumentar el diámetro del trozo. 
BOSQUE 26(2): 107-114, 2005

Evaluación del costo de producción para faenas de aserrío portátil

\section{CUADRO 7}

Rendimientos promedio por clase diamétrica para 1 y 2 pulgadas de espesor. Yields average by diametrical class for 1 and 2 inches of thickness.

\begin{tabular}{|ccccc|}
\hline & \multicolumn{2}{c}{1 pulgada espesor } & \multicolumn{2}{c|}{2 pulgadas espesor } \\
\cline { 2 - 5 } Clase diamétrica & $\mathrm{N}^{\mathrm{o}}$ de trozos & $\begin{array}{c}\text { Rendimiento } \\
\text { pulg/min }\end{array}$ & $\mathrm{N}^{\mathrm{o}}$ de trozos & $\begin{array}{c}\text { Rendimiento } \\
\text { pulg/min }\end{array}$ \\
\hline $20-30$ & 3 & 0,17 & 1 & 0,23 \\
$31-40$ & 1 & 0,34 & 4 & 0,36 \\
$41-50$ & 4 & 0,49 & 6 & 0,54 \\
$51-60$ & 5 & 0,78 & 6 & 0,74 \\
$61-70$ & 9 & 0,63 & 9 & 0,87 \\
$71-80$ & 1 & 0,70 & 3 & 0,94 \\
\hline
\end{tabular}

Análisis de sensibilidad. Para el análisis de sensibilidad se utilizaron los indicadores VAN y TIR, en dos escenarios diferentes de producción, con el fin de observar las tendencias de comportamiento de estos indicadores. La evaluación de ambos escenarios se detalla en el cuadro 8 .

Del análisis de este cuadro se desprende que el proyecto está elaborado sobre la base de antecedentes reales, lo que ha significado realizar estimaciones objetivas y conservadoras. El éxito de estos proyectos no está ligado a la producción en términos de desempeño del equipo, sino más bien a la continuidad del abastecimiento de madera en el tiempo. Igualmente relevante es el precio de mercado de la madera aserrada.

El primer escenario muestra un costo unitario por pulgada alto, donde se cuida el aspecto man- tención y depreciación entre otros ítemes, los que, por lo general, no son considerados por los usuarios, lo que hace que las estimaciones de los beneficios aumenten de manera poco confiable.

\section{CONCLUSIONES}

- El sistema ASEPORT logró predecir adecuadamente los valores para los costos de diferentes productos en una faena determinada, permitiendo con el ajuste de sus valores estimar la situación bajo otras condiciones.

- El simulador permite identificar de manera rápida y detallada los costos fijos y variables de la operación, con una valoración global para la faena.

\section{CUADRO 8}

Evaluación económica bajo dos escenarios de producción.

Economic evaluation under two scenes of production.

\begin{tabular}{|lll|}
\hline & \multicolumn{1}{c|}{ Escenario 1 } & \multicolumn{1}{c|}{ Escenario 2 } \\
\hline Volumen mensual & 4.000 pulgadas en trozo & 6.000 pulgadas en trozo \\
Costo por pulgada estimado & & \\
por el evaluador & US\$ 0,70 & US\$ 0,54 \\
Precio por pulgada aserrada & US\$ 0,80 & US\$ 0,80 \\
Horizonte de evaluación & 6 años & 6 años \\
VAN & US\$ 4.746,89 & US\$ 51.168,24 \\
TIR & $8 \%$ & $54 \%$ \\
\hline
\end{tabular}

Nota: $1 \mathrm{~m}^{3} \mathrm{ssc}=42,4$ pulgadas madereras. 
BOSQUE 26(2): 107-114, 2005

Evaluación del costo de producción para faenas de aserrío portátil

- ASEPORT muestra ser un simulador de costos completo y detallado, que entrega información fiable y útil para estimar la factibilidad económica de realizar una faena determinada.

- El estudio de tiempo constituye una herramienta fundamental para la determinación de los rendimientos y los costos, los cuales se verán fuertemente influenciados de acuerdo con las variaciones experimentadas por los diferentes componentes del ciclo de trabajo.

\section{BIBLIOGRAFIA}

(1) INFOR-CORFO. 1987. Visión tecnológica de la industria forestal chilena. Informe técnico $N^{\circ} 105$. Santiago. Chile. 57 p.

(2) INFOR-CORFO. 1989. Análisis y diagnóstico de procesos industriales de transformación mecánica de la madera. Parte III: estudio de la utilización del tiempo de tra- bajo en plantas de aserrío. Informe técnico No 119 . Santiago, Chile. 57 p.

(3) SOMERVILLE, A. 1988. Recouping the pruning investment. New Zealand Forestry (5): 26-27.

(4) COWN, D.J., D.L. MCCONCHIE, C. Treloar 1984 Timber recovery from pruned Pinus radiata butt logs at Mangatu: Effect of log sweep. New Zealand Journal of Forestry Science, 14(1): 109-123.

(5) BAUMGARTNER, W. 1996. Rendimiento óptimo de las fases aserrío-elaboración en aserraderos Aragón S.A. Tesis Ing. Forestal. Talca, Chile. Universidad de Talca, Facultad de Recursos Naturales. 79 p.

(6) UDDEHOLM. 1996. How to increase profit in bandsawing. Practical models for increased lumber recovery. Uddeholm Strip Steel AB, Sweden. 40 p.

(7) DENIG, J. 1993. Small Sawmill Handbook. Doing it right and making money. Miller Freeman Inc. San Francisco, CA. 182 p.

(8) INFOR. 1971. Estudio de madereo con caballos. Informe técnico $N^{\circ}$ 39. Santiago. Chile. 54 p.

(9) INFOR. 1972. Madereo mecanizado con tractor forestal articulado. Informe técnico $\mathrm{N}^{\circ}$ 40. Santiago. Chile. s/p.

(10) BIAGGIO, G. 1987. Distribución del valor de la madera aserrada a lo largo del fuste en árboles de Pino insigne (Pinus radiata D. Don). Tesis Ing. Forestal. Valdivia, Chile. Universidad Austral de Chile, Facultad de Ciencias Forestales. 60 p. 